\title{
Acknowledgment to Reviewers of Psychiatry International in 2021
}

\section{Psychiatry International Editorial Office}

Citation: Psychiatry International

Editorial Office. Acknowledgment to

Reviewers of Psychiatry International in 2021. Psychiatry Int. 2022, 3, 65-66. https://doi.org/10.3390/

psychiatryint3010006

Published: 29 January 2022

Publisher's Note: MDPI stays neutral with regard to jurisdictional claims in published maps and institutional affiliations.

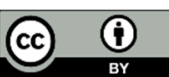

Copyright: () 2022 by the author. Licensee MDPI, Basel, Switzerland. This article is an open access article distributed under the terms and conditions of the Creative Commons Attribution (CC BY) license (https://creativecommons.org/licenses/by/4.0/)

MDPI AG, St. Alban-Anlage 66, 4052 Basel, Switzerland

Rigorous peer-reviews are the basis of high-quality academic publishing. Thanks to the great efforts of our reviewers, Psychiatry International was able to maintain its standards for the high quality of its published papers. Thanks to the contribution of our reviewers, in 2021, the median time to first decision was 18.5 days and the median time to publication was 49.5 days. The editors would like to extend their gratitude and recognition to the following reviewers for their precious time and dedication, regardless of whether the papers they reviewed were finally published:

Almeida, Fernando

Angeletti, Gloria

Bagley, Christopher Adam

Barrios-Fernández, Sabina

Bassett, Darryl

Battaglia, Simone

Bild, Walther

Blicharski, Tomasz

Borzikowsky, Christoph

Byeon, Haewon

Chhatbar, Pratik Yashvant

Cuadradoa, Antonio

De Berardis, Domenico

De La Cruz, Milagros Hidalgo

De Stefano, Rosa

Del Casale, Antonio

Díez Ruiz, Fernando

Donato, Rosario Francesco

Donkor, Prince

Dorotea, Muck-Seler

Dubol, Manon

Dziurkowska, Ewelina

Englund, Tessa R.

Fernandes, Patrícia Raquel Silva

Fernández Carrasco, Francisco Javier

Führer, Amand

Gonzalez-Rodriguez, Alexandre

Gupta, Nilaksh

Gupta, Snehil

Himmerich, Hubertus

Hsu, Mei-Chi

Jannini, Tommaso B.
Javier Jiménez-Jiménez, Felix

Jones, Stephen

Kasai, Shinya

Kepalaite, Albina

Kolcsár, Melinda

Kotsis, Konstantinos

Kotzalidis, Giorgio D.

Krysta, Krzysztof

Kusljic, Snezana

Liutsko, Liudmila

Magnavita, Nicola

Marques, Adilson

Martos Martínez, África

Mawson, Anthony

Mesko, Maja

Mickael, Michel-Edwar

Miralles, Sandra

Mosteller, Jill R.

$\mathrm{Ng}$, Qin Xiang

Niedermoser, Daryl Wayne

Nieto Escámez, Francisco

Novikova, Irina

Pacini, Matteo

Patel, Dilip R.

Patwardhan, Avinash R.

Peerenboom, Peter Bob

Perez, Francisco

Peritogiannis, Vaios

Pozo-Rico, Teresa

Rahman, Hadiar

Raju, Hariharan

Ramirez, Paul Michael 
Rapee, Ronald M.

Rodríguez, Francisco

Rudaz, Myriam

Rudnick, Abraham

Ruggieri, Ruggero Andrisano

Salavera, Carlos

Seeman, Mary V.

Serafini, Gianluca

Simonneau, Michel

Stoyanov, Drozdstoj St.

Styk, Wojciech

Tang, Shifang

Thompson, Miles D.
Tiziana, Mancini

Tomaszek, Katarzyna

Toribio-Mateas, Miguel

Trenado, Carlos

Tripathi, Ashutosh

Vargas-Román, Keyla

Wei, Zheng Zachory

Wu, Yang

$\mathrm{Xi}$, Zheng-Xiong

Yadav, Barkha

Zaręba, Kornelia

Zhang, Siwei

Ziomkiewicz-Wichary, Anna 